\title{
Mise au point de microtests \\ "phosphatase alcaline » et "peroxydase " \\ pour le contrôle de la pasteurisation du lait de vache
}

\author{
par \\ D. MONGET et P. LAVIOLETTE \\ Laboratoire de Biologie - 406 \\ Institut National des Sciences Appliquées \\ 69621 Villeurbanne cedex
}

\section{INTRODUCTION}

L'étude des enzymes dans le secteur laitier s'est développée avec l'introduction de la pasteurisation qui nécessite la mise en œuvre de méthodes rapides et fiables pour distinguer un lait cru d'un lait chauffé. Il est en effet indispensable de vérifier si la température de pasteurisation a été correctement respectée et éventuellement si du lait cru n'a pas été ajouté à du lait pasteurisé à la suite d'une erreur de manipulation ou d'un incident technique.

Parmi les enzymes que contient le lait, la peroxydase (Pien, 1945), et la phosphatase alcaline (Hansen et al., 1953 ; Jacquet et Villette, 1954 ; Alais, 1975 ; Linden et Ged, 1976) ont été les plus étudiées et sont utilisées depuis plus de 30 ans pour le contrôle de la pasteurisation. En effet, si la peroxydase est un peu moins thermolabile que la phosphatase alcaline, leur dénaturation par la chaleur exige des conditions de durée et température qui encadrent étroitement celles que requiert la pasteurisation. Tout lait pasteurisé correctement doit avoir une phosphatase alcaline négative.

En France, les normes officielles de pasteurisation sont les suivantes :

- pour la pasteurisation : « $63^{\circ} \mathrm{C}$ pendant $30 \mathrm{mn} »$ (pasteurisation basse), ou $95^{\circ} \mathrm{C}$ instantanément. En fait dans la pratique, une température intermédiaire est utilisée, généralement au moins $80^{\circ} \mathrm{C}$ pendant quelques secondes (pasteurisation haute). Une telle pasteurisation doit détruire totalement la peroxydase ;

- pour la pasteurisation employée pour obtenir un lait dit de " haute qualité » : $72^{\circ} \mathrm{C}$ à $75^{\circ} \mathrm{C}$ pendant $15 \mathrm{~s}$. 
Bien que peu répandu, ce dernier mode de pasteurisation moins poussée, associé à un contrôle bactériologique très strict (30 000 germes $/ \mathrm{ml}$ au maximum), a l'avantage de préserver les qualités organoleptiques et la majeure partie des éléments nutritifs du lait, tout en assurant la destruction quasi-totale des micro-organismes pathogènes qu'il peut renfermer.

Par arrêté du 2 juin 1955, les méthodes officielles de dosage sont :

- pour la phosphatase alcaline, la méthode de Sanders et Sager (1947) dont le principe est la mesure colorimétrique du phénol libéré par l'enzyme à partir du phénylphosphate ;

- pour la peroxydase, la méthode de Dupouy (voir Pien, 1945), dont le principe est la mesure colorimétrique de l'oxydation chimique du gaïacol sous l'action de l'oxygène libéré par l'enzyme à partir de l'eau oxygénée.

Devant l'importance de ces deux enzymes dans le domaine laitier, nous nous sommes proposés de miniaturiser à partir du dispositif API ZYM (Monget, 1975), une méthode de dosage pour chacune d'elles, qui demande une mise en œuvre la plus simple possible pour l'utilisateur. Pour juger de l'efficacité de ces deux microtests, nous comparerons leur sensibilité à celle des méthodes officielles, en les éprouvant sur :

- des échantillons de laits de vache :

cru ;

pasteurisé à des degrés divers ;

UHT ;

- des mélanges de laits de vache cru et pasteurisé.

\section{MATERIEL ET METHODES}

\section{Réalisation des tests API ZYM « Phosphatase alcaline " et "Peroxydase "}

\section{Phosphatase alcaline}

Principe : la phosphatase alcaline hydrolyse la phénolphtaléine monophosphate et libère dans le milieu la phénolphtaléine qui vire de l'incolore au rose lorsque le $\mathrm{pH}$ est supérieur à 10 .

On prépare un tampon carbonate-bicarbonate $0,3 \mathrm{M}$ pH 10,5 en dissolvant dans 11 d'eau :

$$
\begin{array}{r}
25,81 \mathrm{~g} \text { de carbonate de sodium; } \\
4,83 \mathrm{~g} \text { de bicarbonate de sodium; }
\end{array}
$$

- $113 \mathrm{mg}$ de phénolphtaléine monophosphate sont alors dissous dans $100 \mathrm{ml}$ du tampon précédent et $40 \mu \mathrm{l}$ de cette solution, soit 100 nanomoles de substrat, sont inoculés dans des cupules API ZYM. 
Remarque : la molarité élevée du tampon est nécessaire pour maintenir un $\mathrm{pH}$ supérieur à 10 dans la cupule. Sans cette précaution, le lait qui possède un fort pouvoir tampon acide abaisse le $\mathrm{pH}$ audessous de 10 et empêche la mise en évidence colorimétrique directe de l'enzyme.

Peroxydase (Monget, 1978)

Principe : en présence de peroxydase et d'eau oxygénée, l'O-tolidine se transforme en un produit d'oxydation fortement coloré en bleu :

- 71,2 mg de dichlorhydrate d'O-tolidine et $1 \mathrm{~g}$ de polyvinylpyrrolidone, utilisé comme agent dispersant et mouillant, sont dissous dans $100 \mathrm{ml}$ de tampon acide citrique / citrate de sodium $0,1 \mathrm{M}$, $\mathrm{pH} 4,0$. L'inoculation des cupules est réalisée avec $40 \mu \mathrm{l}$ de cette solution, soit 100 nanomoles d'O-tolidine.

Les tests "Phosphatase alcaline » et "Peroxydase " sont séchés à $37^{\circ} \mathrm{C}$ pendant $4 \mathrm{~h}$ à l'obscurité, puis conservés dans un récipient opaque à la lumière, à température ambiante et sous déshydratant.

Pour faciliter leur utilisation, nous avons associé les deux microtests au sein de bandelettes détachables à deux cupules, regroupées par vingt-cinq sur une même galerie.

\section{Pratique des tests}

\begin{tabular}{|c|c|}
\hline Phosphatase alcaline & Peroxydase \\
\hline $\begin{array}{l}\text { deux gouttes de lait } \\
\text { incubation } 30 \mathrm{mn} \text { ou plus } \\
\text { coloration rose } \\
\text { en présence de l'enzyme }\end{array}$ & $\begin{array}{l}\text { une goutte } \mathrm{H}_{2} \mathrm{O}_{2} \text { diluée } \\
\text { apparition rapide d'une coloration } \\
\text { bleue si la réaction est positive }\end{array}$ \\
\hline
\end{tabular}

Remarques:

- les tests sont généralement effectués à température ambiante. La réaction de la peroxydase est particulièrement instable au-delà de $30^{\circ} \mathrm{C}-32^{\circ} \mathrm{C}$; 


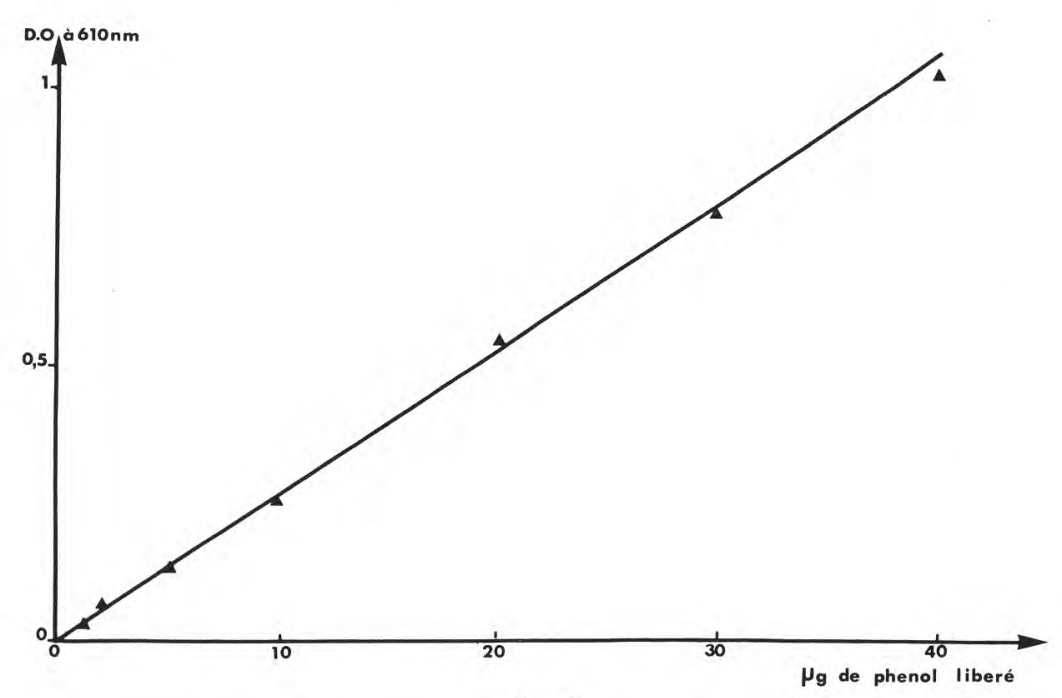

fig. 1

Courbe d'étalonnage pour la recherche de la phosphatase alcaline par la méthode de Sanders et Sager (1947).

- il est important de respecter la quantité d'eau oxygénée ajoutée dans le test " peroxydase ". Pour des concentrations trop élevées, l'enzyme est inhibée ;

- si le test «phosphatase alcaline " doit se prolonger au-delà de $1 \mathrm{~h}$, prévoir une boîte d'incubation faisant office de chambre humide.

\section{RESULTATS}

Nous avons essentiellement comparé la sensibilité des deux microtests que nous proposons pour le contrôle de la pasteurisation à celle des méthodes officielles.

La figure 1 représente la courbe d'étalonnage du dosage de la phosphatase alcaline par la méthode de Sanders et Sager (1947).

L'épreuve à la peroxydase par la méthode de Dupouy fournit seulement une estimation semi-quantitative de l'activité de l'enzyme.

\section{Influence du chauffage}

Le tableau 1 regroupe l'ensemble des résultats que nous avons obtenus à partir :

- de lait cru ; 


\section{$T A B L E A U 1$}

Etude comparée de la recherche de la phosphatase alcaline et de la peroxydase de laits de vache crus et pasteurisés, par les méthodes officielles et les microtests API ZYM

\begin{tabular}{|c|c|c|c|c|c|}
\hline & & \multicolumn{2}{|c|}{ Phosphatase alcaline } & \multicolumn{2}{|c|}{ Peroxydase } \\
\hline & & $\begin{array}{l}\text { Méthode de } \\
\text { Sanders et Sager }\end{array}$ & $\begin{array}{l}\text { Microtest } \\
\text { API ZYM }\end{array}$ & $\begin{array}{l}\text { Méthode de } \\
\text { Dupouy }\end{array}$ & $\begin{array}{l}\text { Microtest } \\
\text { API ZYM }\end{array}$ \\
\hline \multicolumn{2}{|c|}{ Lait cru (dix échantillons) } & $>40(1)$ & $+++++(2)$ & $++++(3)$ & $+++++(3)$ \\
\hline Lait pasteurisé $20 \mathrm{~s}$ à & $\begin{array}{ll}60^{\circ} & \mathrm{C} \\
62^{\circ} & \mathrm{C} \\
64^{\circ} & \mathrm{C} \\
66^{\circ} & \mathrm{C} \\
68^{\circ} & \mathrm{C} \\
70^{\circ} & \mathrm{C} \\
72^{\circ} & \mathrm{C} \\
74^{\circ} & \mathrm{C} \\
76^{\circ} & \mathrm{C} \\
78^{\circ} & \mathrm{C} \\
80^{\circ} & \mathrm{C} \\
82^{\circ} & \mathrm{C}\end{array}$ & $\begin{aligned} &> 40 \\
&> 40 \\
&> 40 \\
&> 40 \\
& 24,8 \\
& 5,7 \\
& 2,1 \\
& 1,9 \\
& 1,7 \\
& 1,5 \\
&< 1 \\
&< 1\end{aligned}$ & $\begin{array}{c}+++++ \\
+++++ \\
+++++ \\
+++ \\
++ \\
\mathrm{t}(+2 \mathrm{~h}) \\
0 \\
0 \\
0 \\
0 \\
0 \\
0\end{array}$ & $\begin{array}{c}+++++ \\
+++++ \\
+++++ \\
+++++ \\
+++++ \\
+++++ \\
+++++ \\
+++ \\
++ \\
+ \\
0 \\
0\end{array}$ & $\begin{array}{c}+++++ \\
+++++ \\
+++++ \\
+++++ \\
+++++ \\
+++++ \\
+++++ \\
+++++ \\
+++++ \\
+++ \\
+ \\
0\end{array}$ \\
\hline \multicolumn{2}{|c|}{ Lait pasteurisé du commerce (cinq échantillons) } & $<1$ & 0 & 0 & 0 \\
\hline \multicolumn{2}{|c|}{ Lait U.H.T. (trois échantillons) } & $<1$ & 0 & 0 & 0 \\
\hline
\end{tabular}

(1) En $\mu \mathrm{g}$ de phénol libéré / $0,5 \mathrm{ml}$ de lait en $1 \mathrm{~h}$ à $37^{\circ} \mathrm{C}$.

(2) Note de 0 à +++++ , selon l'intensité de la coloration, après une incubation de $1 \mathrm{~h}$ à température ambiante. $\mathrm{t}=\operatorname{trace}(0<\mathrm{t}<+)$.

(3) Note de 0 à +++++ , selon l'intensité de la coloration, après une incubation de $10 \mathrm{mn}$ à température ambiante. 
- de lait cru que nous avons chauffé entre $60^{\circ} \mathrm{C}$ et $82^{\circ} \mathrm{C}$ au bain-marie pendant $20 \mathrm{~s}$ dans un tube capillaire en verre très fin (diamètre intérieur $2 \mathrm{~mm}$, épaisseur de la paroi $0,2 \mathrm{~mm}$ ), à défaut d'avoir pu disposer d'un pasteurisateur expérimental ; U.H.T.

- de laits du commerce pasteurisés (pasteurisation haute) et

Ce tableau indique clairement la différence de thermolabilité entre la phosphatase alcaline et la peroxydase : après un chauffage de $20 \mathrm{~s}$ la première enzyme est dénaturée vers $70^{\circ} \mathrm{C}$ et la seconde vers $80^{\circ} \mathrm{C}$.

Selon les normes, on considère qu'un lait est bien pasteurisé lorsque la quantité de phénol libéré par la phosphatase alcaline dans la méthode de Sanders et Sager (1947) ne dépasse pas $2 \mu \mathrm{g}$ pour 0,5 ml de lait. Ainsi dans le cas présent, en utilisant un tube capillaire, la pasteurisation est correcte, pour un temps de chauffage de $20 \mathrm{~s}$, si la température est au moins égale à $72^{\circ} \mathrm{C}$.

La zone de température $72^{\circ} \mathrm{C}-75^{\circ} \mathrm{C}$, pour un temps de chauffage de $20 \mathrm{~s}$, correspond à une pasteurisation dite de " haute qualité » pour laquelle la phosphatase alcaline est détruite (quantité de phénol libéré inférieure à $2 \mu \mathrm{g} / 0,5 \mathrm{ml}$ de lait), alors que la peroxydase demeure active.

La pasteurisation industrielle la plus couramment effectuée (pasteurisation haute) dénature la peroxydase et a fortiori la phosphatase alcaline. Ce résultat est obtenu expérimentalement en chauffant le lait au-delà de $81^{\circ} \mathrm{C}$ pendant $20 \mathrm{~s}$.

Ainsi, en raison des propriétés thermolabiles différentes de ces deux enzymes, il est très facile de distinguer les deux types de pasteurisation :

\begin{tabular}{l|c|c}
\hline & Phosphatase alcaline & Peroxydase \\
\hline Pasteurisation classique & détruite \\
Pasteurisation de " haute qualité » & détruite & détruite \\
& active \\
\hline
\end{tabular}

Pour l'épreuve à la phosphatase alcaline, la méthode API ZYM (1 h d'incubation, température ambiante) et celle de Sanders et Sager (1947) ont une sensibilité voisine. Bien que le seuil minimal de détection de $1^{\prime}$ enzyme $\left(70^{\circ} \mathrm{C}\right.$ pendant $\left.20 \mathrm{~s}\right)$ soit sans aucun doute plus facile à déterminer au moyen d'un photomètre que visuellement, nos résultats montrent qu'un écart de $1^{\circ} \mathrm{C}$ en deçà de celui-ci est parfaitement 
repérable à l'aide du microtest API ZYM et donc qu'il est possible d'estimer sans risque d'erreur le seuil de $70^{\circ} \mathrm{C}$ au degré près. Ce dernier peut être apprécié sans difficulté lorsque le microtest est pratiqué en $2 \mathrm{~h}$. Cette durée correspond au temps total nécessaire pour doser la phosphatase alcaline par la méthode de Sanders et Sager (1947).

Le dosage de la peroxydase apparaît un peu plus sensible avec le microtest API ZYM que par la méthode de Dupouy, puisque pour un chauffage de $20 \mathrm{~s}$, les seuils de détection de l'enzyme se situent respectivement à $80^{\circ} \mathrm{C}$ et $78^{\circ} \mathrm{C}$.

\section{Adjonction de lait cru à du lait pasteurisé à haute température}

Nous avons effectué différents mélanges de lait cru et de lait pasteurisé du commerce, dans le but de rechercher la concentration minimale détectable de lait cru qui peut être ajoutée accidentellement à du lait déjà traité par la chaleur (tab. 2).

Il ressort qu'avec les microtests API ZYM, tout comme avec les méthodes officielles, l'épreuve à la phosphatase alcaline est mieux adaptée que celle de la peroxydase pour la mise en évidence de mélanges à faibles taux de lait cru.

La méthode API ZYM pour la recherche de la phosphatase alcaline (incubation $1 \mathrm{~h}$ à température ambiante) apparaît un peu moins sensible que celle de Sanders et Sager (1947), les seuils de détection étant respectivement de 1 p. 100 et de 0,5 p.100. Les résultats deviennent par contre comparables si l'incubation du microtest est réalisée à $37^{\circ} \mathrm{C}$. Notons qu'en prolongeant la durée de la réaction enzymatique, on améliore nettement les performances de celui-ci, puisqu'après une nuit d'incubation ( $16 \mathrm{~h})$ à température ambiante, on peut détecter jusqu'à 0,1 p. 100 d'apport de lait cru dans le lait pasteurisé.

Alors que le seuil de 5 p. 100 est parfaitement lisible avec le test API ZYM "Peroxydase ", 10 p. 100 constitue la limite de positivité de la méthode de Dupouy. Ce résultat confirme que le test API ZYM est plus sensible que la technique officielle.

\section{DISCUSSION ET CONCLUSIONS}

L'utilisation de techniques lourdes et délicates à mettre en œuvre constitue à l'heure actuelle un gros handicap pour l'industrie laitière, alors que les contrôles systématiques deviennent de plus en plus nombreux. Nous prendrons comme seul exemple l'épreuve de la phosphatase alcaline par la méthode officielle de Sanders et Sager (1947). Bien que pratiquée en routine dans tous les laboratoires, elle n'en reste pas moins une méthode laborieuse, qui demande la préparation de six réactifs au minimum et surtout un grand nombre de manipula- 
TABLEAU 2

Sensibilité de la recherche de la phosphatase alcaline et de la peroxydase dans le lait de vache, en cas d'adjonction par accident de lait cru dans du lait pasteurisé du commerce (pasteurisation haute)

\begin{tabular}{|c|c|c|c|c|c|}
\hline & \multicolumn{3}{|c|}{ Phosphatase alcaline } & \multicolumn{2}{|c|}{ Peroxydase } \\
\hline & $\begin{array}{c}\text { Méthode de } \\
\text { Sanders et Sager }\end{array}$ & $\begin{array}{l}\text { Microtest } \\
1 \mathrm{~h}\end{array}$ & $\begin{array}{l}\text { I ZYM } \\
16 \mathrm{~h}\end{array}$ & $\begin{array}{l}\text { Méthode de } \\
\text { Dupouy }\end{array}$ & $\begin{array}{l}\text { Microtest } \\
\text { API ZYM }\end{array}$ \\
\hline Lait pasteurisé du commerce (témoin) & $0(1)$ & $0(2)$ & $0(2)$ & $0(3)$ & $0(3)$ \\
\hline Lait cru (témoin) & $>40$ & ++++ & ++++ & ++++ & ++++ \\
\hline Lait pasteurisé +10 p. 100 lait cru & $>40$ & +++ & ++++ & + & ++ \\
\hline Lait pasteurisé +5 p. 100 lait cru & $>40$ & ++ & +++ & 0 & + \\
\hline Lait pasteurisé +2 p. 100 lait cru & 27,2 & $+\left(++\right.$ à $\left.37^{\circ} \mathrm{C}\right)$ & +++ & 0 & 0 \\
\hline Lait pasteurisé +1 p. 100 lait cru & 12,0 & $\mathrm{t}\left(+\mathrm{à} 37^{\circ} \mathrm{C}\right)$ & ++ & 0 & 0 \\
\hline Lait pasteurisé $+0,5$ p. 100 lait cru & 5,4 & $0\left(\mathrm{t}\right.$ à $\left.37^{\circ} \mathrm{C}\right)$ & + & 0 & 0 \\
\hline Lait pasteurisé $+0,2$ p. 100 lait cru & 1,2 & 0 & + & 0 & 0 \\
\hline Lait pasteurisé $+0,1$ p. 100 lait cru & $<1$ & 0 & $\mathrm{t}$ & 0 & 0 \\
\hline
\end{tabular}

(1) En ug de phénol libéré / 0,5 $\mathrm{ml}$ de lait, en $1 \mathrm{~h}$ à $37^{\circ} \mathrm{C}$.

(2) Note de 0 à +++++ , selon l'intensité de la coloration. $t=$ trace $(0<t<1)$ (incubation à température ambiante).

(3) Note de 0 à +++++ , selon l'intensité de la coloration, après une incubation de $10 \mathrm{mn}$ à température ambiante. 
tions s'échelonnant sur une durée de 2 h. Aschaffenburg et Mullen (1949) ont proposé de simplifier la méthode par l'emploi du paranitrophénylphosphate comme substrat.

Pour la recherche et le dosage des enzymes, Jacquet et al. (1975) sont persuadés que la méthode API ZYM, à condition de ne retenir que les caractères les plus intéressants, doit rendre de nombreux services dans le domaine laitier, en raison précisément de sa simplicité d'utilisation. La surveillance de la pasteurisation du lait, examen effectué en grande série dans les laboratoires spécialisés, devrait être une des applications les plus immédiates de la microméthode. C'est pourquoi, nous avons sélectionné et adapté les tests « phosphatase alcaline " et "peroxydase », utilisés pour ce type de contrôle. De par leur conception, le nombre d'opérations à la charge de l'utilisateur se limite à l'addition de lait et d'eau oxygénée diluée. Il est difficile d'imaginer méthode plus simple.

Pour l'épreuve de la phosphatase alcaline, le choix de la phénolphtaléine monophosphate comme substrat nous a paru s'imposer. D'une part, la phénolphtaléine, rose en milieu alcalin, est beaucoup plus visualisable que le paranitrophénol, coloré en jaune ; d'autre part, l'utilisation du 2-naphtylphosphate nécessite l'emploi de deux réactifs, dont le Fast Blue $\mathrm{BB}$ pour révéler le 2-naphtol libéré par l'enzyme.

Notre étude comparative a montré qu'il existe une bonne concordance entre les deux microtests « phosphatase alcaline » et " peroxydase " que nous avons mis au point et les méthodes officielles de dosage de ces deux enzymes. Pour le contrôle de la pasteurisation, les caractères API ZYM offrent une sensibilité voisine de celle des techniques classiques. Dans un tube capillaire, il est évidemment impossible de reproduire le traitement physique auquel est soumis le lait au cours de la pasteurisation industrielle. Aussi, l'utilisation d'un bain-marie peut-elle être sujette à critique. La dénaturation de la phosphatase alcaline à $72^{\circ} \mathrm{C}$ montre cependant qu'une incubation de $20 \mathrm{~s}$, telle que nous la pratiquons, se rapproche des conditions de la pasteurisation de « haute qualité ». On peut estimer que quelques secondes suffisent pour que la température du lait à l'intérieur du tube capillaire s'équilibre avec celle du bain-marie.

La contamination de lait chauffé à haute température par du lait cru est un accident qui se produit de temps à autre dans les laiteries à la suite d'une fuite dans un pasteurisateur. Le test API ZYM « phosphatase alcaline » et la méthode de Sanders et Sager (1947) ne permettent pas de mettre en évidence moins de 0,5 p. 100 d'apport de lait cru, après $1 \mathrm{~h}$ d'incubation en présence du substrat. Avec la méthode d'Aschaffenburg et Mullen (1949) ce seuil est de 1 p. 100 à 5 p. 100 dans les mêmes conditions. Par contre, lorsque cela s'avère nécessaire, il est possible d'augmenter la sensibilité du test API ZYM sans modifier le protocole opératoire, en prolongeant simplement la durée de la réaction enzymatique. Le seuil de 0,1 p. 100 peut ainsi être atteint 
en 16 h d'incubation, alors que d'après Ainas (1975), il est de 0,2 p. 100 en $24 \mathrm{~h}$ par la méthode de diffusion en gélose et de 0,3 p. 100 par celle de Aschaffenburg et Mullen (1949). Une longue incubation ne constitue pas forcément un inconvénient majeur, dans la mesure où elle peut être réalisée la nuit.

Outre la simplicité, la rapidité et la sensibilité qu'offre le dispositif mis au point, nous nous devons de signaler également deux avantages non négligeables : son utilisation ne nécessite aucun équipement de laboratoire et supprime le problème du recyclage de la verrerie, notamment lors de l'épreuve à la phosphatase alcaline. Ainsi, toutes les conditions sont réunies pour que ce « kit » prêt à l'emploi trouve facilement une place sur le marché aux côtés des méthodes traditionnelles.

\section{Rés u m é}

Deux microtests colorimétriques, simples et rapides, ont été mis au point pour la recherche de la phosphatase alcaline et de la peroxydase dans le lait de vache, en utilisant respectivement comme substrats chromogènes la phénolphtaléine monophosphate et l'O-tolidine.

Ces tests présentent une sensibilité comparable à celle des méthodes officielles, aussi bien pour le contrôle de la pasteurisation que pour la détection de lait cru ajouté à du lait pasteurisé.

\section{S u $\mathrm{m}$ m a ry}

PHOSPHATASE ALCALINE AND PEROXYDASE MICROTESTS FOR THE CONTROL OF PASTEURIZATION OF COW MILK

Two simple, quick and reliable colorimetric microtests were fitted to check alcaline phosphatase and peroxydase in cow milk, with respectively the phenolphtalein monophosphate and the O-tolidine as chromogenic substrates.

These tests allow a sensitiveness similar to the other official methods used either for the control of pasteurization or for the detection of raw milk added to the pasteurized one.

\section{Remerciements}

Nous tenons à remercier M. AUCLAIR, Directeur de la Station de Technologie laitière (I.N.R.A.) de Jouy-en-Josas, qui a bien voulu procéder à des essais de notre matériel et relire notre manuscrit.

Nous remercions également M. GELIN, Conseiller Scientifique et Technique du Groupe SCOFF, qui nous a procuré tous les documents permettant la comparaison des résultats de notre méthode avec les méthodes officielles. 


\section{Bibliographie}

AInas (M.) (1975). - Contribution à l'étude des cellules du lait et de leurs activités. Thèse de $3^{e}$ cycle, Université de Caen, p. 88.

Alais (C.) (1975). - Science du lait. 3e édition, Sep, Paris, 216-224.

Aschaffenburg (R.) and Mullen (J. E. C.) (1949). - A rapid and simple phosphatase test for milk. J. Dairy Res., 16, 58-67.

Hanser (S. A.), Wood (F. W.) and THORnton (H. R.) (1953). - Phosphatase inactivation in pasteurization of milk. Can. J. Technol., 31, 240-249.

JAcQuet (J.) et Villette (O.) (1954). - Etude sur les phosphatases du lait de vache. Bull. Acad. Vét., 27, 429-433.

JACOUET (J.), Seng-Huor (T.) et Ainas (M.) (1975). - Méthodes de recherches simples de quelques enzymes. Applications. Bull. Acad. Vét., 48, 467-472.

Linden (G.) et Ged (J.) (1976). - Activité, polymorphisme et origine de la phosphatase alcaline dans le lait. Milchwissenschaft, 31, 724-728.

MoNGET (D.) (1975). - Différence d'activités enzymatiques entre deux lignées cellulaires d'insectes : Antheraea eucalypti et Malacosoma disstria, (Lepidoptera). C.R. Acad. Sci., 281, 651-654, série D.

Monget (D.) (1978). - L'ortho-tolidine : un révélateur plus sensible de la peroxydase dans la méthode immuno-enzymatique Elisa. Ann. Biol. Clin. (à paraître).

PIEN (J.) (1945). - Le contrôle de la pasteurisation du lait et de la crème. Le Lait, 25, 311-320.

SANDERS (G. P.), SAGer (O. S.) (1947). - Phosphatase test for various dairy products, J. Dairy Sci., 30, 909-920. 